Prevalence of Endocervical Chlamydia trachomatis Infection and

\title{
Related Risk Factors Among Women Attending Gynecology Clinic of Birjand University of Medical Sciences, East of Iran
}

\author{
Nahid Gahanbarzade (iD ${ }^{1}$, Elham Ramazani ${ }^{2}$, Masoud Yousefi ${ }^{2}$, Mahmoud Zardast ${ }^{2}$ and Majid \\ Zare-Bidaki (iD) ${ }^{2, *}$ \\ ${ }^{1}$ Department of Gynecology and Obstetrics, Medical Faculty, Birjand University of Medical Sciences, Birjand, Iran \\ ${ }^{2}$ Infectious Diseases Research Center, Birjand University of Medical Sciences, Birjand, Iran \\ "Corresponding author: Infectious Diseases Research Center, Birjand University of Medical Sciences, Birjand, Iran. Email: m.zare@live.co.uk \\ Received 2020 October 26; Revised 2021 May 03; Accepted 2021 May 09.
}

\begin{abstract}
Background: Chlamydia trachomatis is one of the most common sexually transmitted infections in the world. However, there is no detailed information on its incidence, especially in developing countries where routine laboratory diagnosis is unavailable.

Objectives: This study aimed to investigate the prevalence of endocervical C. trachomatis infection and related risk factors among women attending the University Gynecology Clinic of Birjand, East of Iran.

Methods: This cross-sectional study was conducted on 195 women attending the University Gynecology Clinic in South Khorasan, Birjand. Endocervical sampling was performed in a lithotomy position using a sterile brush. Identification of $C$. trachomatis was performed by real-time PCR method using GeneProof C. trachomatis PCR kit. Data on socio-demography and potential risk factors for genital infection were analyzed using SPSS software (version 21).

Results: In the study, the prevalence rate of C. trachomatis among women was reported 4.1\% (8/195 subjects). Statistical analysis showed that the rate of $C$. trachomatis infection in women was only statistically related to the history of vaginal infection $(\mathrm{P}=0.001)$. Although there was no statistically significant association between chlamydial infection and age, the highest infection rate was in women less than 30 years old.

Conclusions: Given the relatively significant incidence of $C$. trachomatis infection among women, our findings highlight the importance of routine screening and early diagnosis of $C$. trachomatis to control the infection.
\end{abstract}

Keywords: Chlamydia trachomatis, Infection, Diagnosis, Risk Factors, Real-Time PCR

\section{Background}

Sexually transmitted infections (STIs) are frequent and constitute a significant health problem in almost all countries. Chlamydia trachomatis is one of the most common STIs globally that is the cause of a broad spectrum of human diseases. Conferring to the latest World Health Organization (WHO) reports, the prevalence of worldwide chlamydial infection has progressively increased over the last two decades, and there were 127 million new cases of Chlamydia in 2016 (1-3).

The $C$. trachomatis infection is often asymptomatic in women (about 75\%) and can lead to chronic manifestations. Untreated chlamydial infections may cause pelvic inflammatory disease (PID), ectopic pregnancy, spontaneous abortion, premature delivery, low birth weight, and infertility (4-6). It is noteworthy that the risk factors of C. tra- chomatis infection in women differ by setting and the existence of symptoms. The infection risk factors include age, inconsistent use of barrier contraception, prior sexually transmitted infection, low educational and socioeconomic levels, cervical infection, and polygamous marriage $(6,7)$.

An essential measure for the prevention and chlamydial infection control can be identifying women with asymptomatic or mild endocervical infections and those at increased risk for acquiring this infection $(7,8)$. The Center for Disease Control and Prevention (CDC) recommends annual screening for C. trachomatis infection in all sexually active women younger than 25 years old. Moreover, women older than 25 years and all sexually active men at risk of the infection should also be screened (9).

The conventional laboratory diagnosis of C. trachomatis infection is made through cell culture or antigen de- 
tection. However, the gold-standard techniques for genital Chlamydia infection are now nucleic acid amplification tests (NAATs). Recently, commercially available NAATs methods, such as polymerase chain reaction (PCR) assays with high sensitivity and specificity, have been widely used for detection of $C$. trachomatis infection $(6,10)$.

\section{Objectives}

Given the importance of identifying chlamydial infection among women in developing countries, this study aimed to evaluate the prevalence of endocervical C. trachomatis infection and related risk factors among women attending the Gynecology Clinic, Birjand University of Medical Sciences, East of Iran.

\section{Methods}

\subsection{Study Population and Sampling Procedure}

This cross-sectional study was conducted on 195 women attending the University Gynecology Clinic in Birjand, the capital of South Khorasan province, Iran, during 2018 - 2019. The study was approved by the Ethics Committee of Birjand University of Medical Sciences (IR.BUMS.REC.1397.88), and all enrolled subjects signed informed consent forms. The inclusion criteria were all women with asymptomatic and symptomatic of chlamydial infection attending the gynecology clinic. However, all pregnant and virgin women were excluded from the study. It is noteworthy that the socio-demographic and obstetric-related characteristics of subjects were collected using a structured questionnaire.

Endocervical sampling was performed in a lithotomy position using a sterile brush by the attending gynecologist. Samples were placed into 0.2 M sucrose phosphate buffer (2SP, $\mathrm{pH}=7.2$ ) (Sigma-Aldrich, USA) containing $0.2 \%$ fetal bovine serum (Gibco, USA) and transported to the microbiology laboratory on ice (5).

\subsection{PCR Assay}

The cervical brushes were removed after vortexing, and the samples were used for further processing. According to the manufacturer, DNA extraction was performed using the High Pure PCR Template Preparation Kit (Roche, Germany) from the samples.

In this study, C. trachomatis was detected by TaqMan real-time PCR method using GeneProof $C$. trachomatis PCR kit (GeneProof, Czech Republic). According to the manufacturer's instructions, the kit detects the cryptic plasmid multi-copy sequence and the $16 \mathrm{~S}$ rRNA gene for C. trachomatis. This kit uses ready-to-use master mix contains uracilDNA glycosylase (UNG) and dUTPs eliminating contamination with amplification products. Briefly, thermal cycling conditions for real-time PCR assay were as follows: $37^{\circ} \mathrm{C}$ for 2 minutes and $95^{\circ} \mathrm{C}$ for 10 minutes followed by 45 cycles of $95^{\circ} \mathrm{C}$ for 5 seconds, $60^{\circ} \mathrm{C}$ for 40 seconds, and $72^{\circ} \mathrm{C}$ for 20 seconds. The signal was acquired at $60^{\circ} \mathrm{C}$ during each cycle. Amplification and PCR product detection were performed with the ABI prism 7500 real-time-PCR System (Applied Biosystems, USA).

\subsection{Statistical Analysis}

The data were investigated with the Pearson chi-square and Fisher's exact tests, using SPSS (version 21), to assess the statistical significance of associations between potential variables. P-values of less than 0.05 were considered to be significant.

\section{Results}

A total of 195 women with a mean age of $33.91 \pm 9.84$ (ranging from 17 to 60) years were included in the study.

\subsection{C. trachomatis Infection and Related Risk Factors}

The prevalence of $C$. trachomatis infection was reported in $4.1 \%$ ( 8 patients) of the study population. The sociodemographic features of the women and their relationship with $C$. trachomatis infection are summarized in Table 1. Statistical analysis results revealed that there was no significant association between socio-demographic factors and C. trachomatis infection. It is noteworthy that although there was no statistically significant association between chlamydial infection and age, the highest rate of infection was in women less than 30 years old (Figure 1).

Moreover, the associations of C. trachomatis infection with the clinical and obstetric risk factors are shown in Tables 2 and 3. The most common symptoms in the females were vaginal discharge (71.4\%), lower abdominal pain (71.4\%), dyspareunia (42.8\%), and burning sensation (42.8\%). It is noteworthy that C. trachomatis infection was associated only with a history of vaginal infection ( $\mathrm{P}=$ 0.001). C. trachomatis infection was reported in $9.5 \%$ of cases with a history of vaginal infection vs. $0 \%$ in those with no history.

\section{Discussion}

C. trachomatis infection is one of the most common bacterial sexually transmitted infections (STIs) worldwide, and women carry the significant burden of the disease. The 


\begin{tabular}{|c|c|c|c|}
\hline Item/Status & Positive & Negative & Significance \\
\hline Age (y) & $34.00 \pm 11.63$ & $33.91 \pm 9.80$ & $t=-0.03 ; \mathrm{df}=193 ; \mathrm{P}=0.98$ \\
\hline Occupation & & & $\chi^{2}=1.06 ; \mathrm{df}=1 ; P=0.60$ \\
\hline Housewife & $8(4.6)$ & $165(95.4)$ & \\
\hline Employed & $0(0)$ & $22(100)$ & \\
\hline Education & & & Fisher $=5.12 ; \mathrm{P}=0.07$ \\
\hline Illiterate & $2(16.7)$ & $10(83.3)$ & \\
\hline High school & $4(4.4)$ & $80(95.2)$ & \\
\hline College & $2(2)$ & $97(98)$ & \\
\hline Dwelling & & & $\chi^{2}=1.96 ; \mathrm{df}=1 ; \mathrm{P}=0.23$ \\
\hline Urban & $4(2.9)$ & $136(97.1)$ & \\
\hline Rural & $4(7.3)$ & $51(92.7)$ & \\
\hline Addiction & & & $\chi^{2}=0.13 ; \mathrm{df}=1 ; \mathrm{P}=0.88$ \\
\hline Yes & $0(0)$ & $3(100)$ & \\
\hline No & $8(4.1)$ & $184(95.9)$ & \\
\hline
\end{tabular}

Abbreviation: df, degree of freedom.

${ }^{\mathrm{a}}$ Values are expressed as mean $\pm \mathrm{SD}$ or No. (\%) unless otherwise indicated.

\begin{tabular}{|c|c|c|c|}
\hline Item/Status & Positive (\%) & Negative (\%) & Significance \\
\hline Clinical symptom & & & $\chi^{2}=0.53 ; \mathrm{df}=1 ; \mathrm{P}=0.68$ \\
\hline Yes & $7(4.7)$ & $143(95.3)$ & \\
\hline No & $1(2.2)$ & $44(97.8)$ & \\
\hline Physical examination & & & Fisher $=3.37 ; \mathrm{P}=0.14$ \\
\hline Genital ulcer & $2(10)$ & $18(90)$ & \\
\hline Herpes lesions & $0(0)$ & $40(100)$ & \\
\hline Normal & $6(4.4)$ & $129(95.6)$ & \\
\hline Diabetes mellitus & & & $\chi^{2}=0.17 ; \mathrm{df}=1 ; \mathrm{P}=0.84$ \\
\hline Yes & $0(0)$ & $4(100)$ & \\
\hline No & $8(4.1)$ & $183(95.9)$ & \\
\hline History of antibiotic usage & & & $\chi^{2}=1.23 ; \mathrm{df}=1 ; \mathrm{P}=0.39$ \\
\hline Yes & $0(0)$ & $25(100)$ & \\
\hline No & $8(4.7)$ & $162(95.3)$ & \\
\hline
\end{tabular}

Abbreviation: df, degree of freedom.

WHO's most recent estimates indicate that in 2016 about 127 million new cases of $C$. trachomatis occurred globally. However, there is no detailed information on the incidence of chlamydial infection, especially in developing countries where routine laboratory diagnosis is unavailable (11-13).

The prevalence rate of $C$. trachomatis among the women was reported at $4.1 \%$ in our study. This finding was lower than many other reports in $\operatorname{Iran}(5,7,14,15)$, and other developing countries, such as Ethiopia (18.9\%), Brazil (10.7\%), Serbia (15.4\%), and India $(23 \%)(1,8,12,16)$, but higher than those reported in Jordan (3.9\%), Nigeria (3.5\%), and another study in Iran $(2.4 \%)(6,17,18)$. Discrepancies in C. trachomatis infection rates among women in the studied populations could be explained in terms of age, ethnic group, socio-economic status, and lifestyle; the geographic areas, hygiene, and barrier contraception during 


\begin{tabular}{|c|c|c|c|}
\hline Item/Status & Positive (\%) & Negative (\%) & Significance \\
\hline Number of pregnancy & & & Fisher $=0.57 ; \mathrm{P}=0.80$ \\
\hline Without pregnancy & $1(2.6)$ & $37(97.4)$ & \\
\hline $1-2$ & $3(3.5)$ & $81(96.5)$ & \\
\hline 3 & $4(5.4)$ & $69(94.6)$ & \\
\hline History of abortion & & & $\chi^{2}=0.12 ; \mathrm{df}=1 ; \mathrm{P}=0.63$ \\
\hline Yes & $2(3.8)$ & $50(96.2)$ & \\
\hline No & $6(4.2)$ & $137(95.8)$ & \\
\hline Type of delivery & & & Fisher $=0.59 ; \mathrm{P}=0.79$ \\
\hline Without delivery & $1(2.4)$ & $41(97.6)$ & \\
\hline Vaginal & $6(5.3)$ & $108(94.7)$ & \\
\hline Cesarean & $1(2.6)$ & $38(97.4)$ & \\
\hline Preterm delivery & & & $\chi^{2}=0.15 ; \mathrm{df}=1 ; \mathrm{P}=0.53$ \\
\hline Yes & $1(5.9)$ & $16(94.1)$ & \\
\hline No & $7(3.9)$ & $171(96.1)$ & \\
\hline History of infertility & & & $\chi^{2}=0.01 ; \mathrm{df}=1 ; \mathrm{P}=0.69$ \\
\hline Yes & $1(3.7)$ & $26(96.3)$ & \\
\hline No & $7(4.2)$ & $161(95.8)$ & \\
\hline History of vaginal infection & & & $\chi^{2}=11.02 ; \mathrm{df}=1 ; \mathrm{P}=0.001$ \\
\hline Yes & $8(9.5)$ & $76(90.5)$ & \\
\hline No & $0(0)$ & $111(100)$ & \\
\hline Sexual activity & & & $\chi^{2}=0.09 ; \mathrm{df}=1 ; \mathrm{P}=0.92$ \\
\hline Active & $8(4.1)$ & $185(95.9)$ & \\
\hline Without intercourse & $0(0)$ & $2(100)$ & \\
\hline
\end{tabular}

Abbreviation: df, degree of freedom.

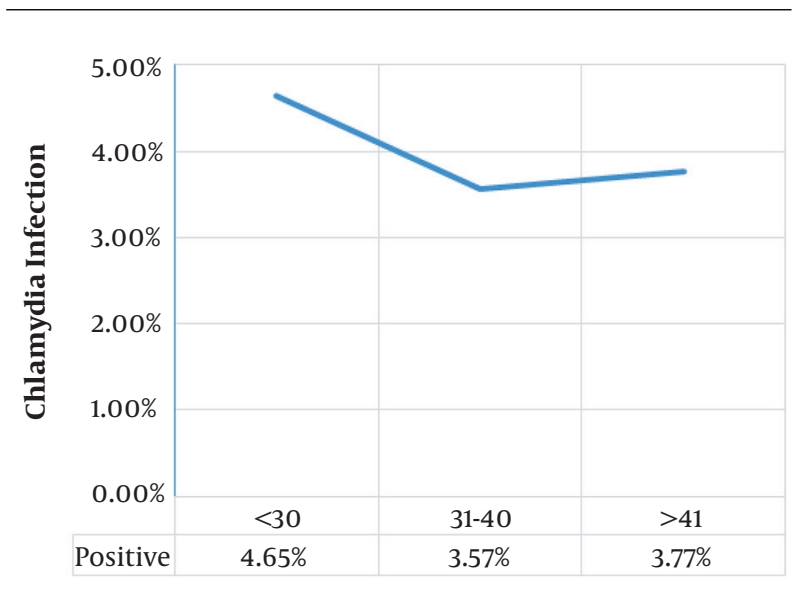

$\overline{\text { Figure 1. Association between C. trachomatis infection and age of women (y) (Fisher }}$ $=0.21, \mathrm{P}=0.99$ ) intercourse, sexual behavior, sampling method, and the diagnostic techniques $(1,7,8)$.

The results of our study showed that there was no significant association between socio-demographic factors and C. trachomatis infection. It is noteworthy that although there was no statistically significant association between chlamydial infection and age, the highest rate of infection was in women less than 30 years old. This finding is consistent with other studies $(6,18-20)$. These age groups all fall within the sexually active, explaining a higher prevalence of STIs among them $(6,20)$. However, it was not statistically significant in our study.

Finally, numerous epidemiological surveys for C. trachomatis in women have identified various associated risk factors for infection $(14,18)$. Interestingly, we found an association between a history of vaginal infection and an increased prevalence of $C$. trachomatis in women. This finding is comparable to those reported by others and suggests that a history of vaginal infection may be the major risk 
factor for chlamydial infection. Kayiira et al. showed that over $36.96 \%$ of the participants with current genital C. trachomatis had a history of genital infection compared to $2.9 \%$ of participants in the non-exposed group (21). Nevertheless, the fact that the sample size did not achieve statistical power can explain the lack of association between $C$. trachomatis infection and other clinical-obstetric risk factors in our study.

\subsection{Conclusions}

Given the relatively significant incidence of $C$. trachomatis infection among women, our findings highlight the status of routine screening and early diagnosis of C. trachomatis to control the infection. However, further studies with a larger sample size and more focused on different high-risk groups are needed to prevent and control the infection. Analysis of the results showed an association between a history of vaginal infection and an increased prevalence of $C$. trachomatis in women, but further studies are needed to assess the correlation between different risk factors and $C$. trachomatis infection.

\section{Acknowledgments}

This research was supported by Birjand University of Medical Sciences, Birjand, Iran (grant number: 455409).

\section{Footnotes}

Authors' Contribution: ER and MZ collected the data and provided the first reports. NGH and MZB designed and supervised the research. MY interpreted the analysis and wrote the final manuscript.

Conflict of Interests: The authors declare that they have no conflict of interest.

Ethical Approval: The study was approved by the Ethics Committee of Birjand University of Medical Sciences (IR.BUMS.REC.1397.88).

Funding/Support: This research was supported by Birjand University of Medical Sciences, Birjand, Iran (grant number: 455409).

Informed Consent: All enrolled subjects signed informed consent forms.

\section{References}

1. Borges JB, Marchesini AC, Stefani LF, Belintani MV, Santos TA. Prevalence of Chlamydia trachomatis infection among women seen at the lower genital tract pathology clinic, Jundiai School of Medicine, Brazil. Einstein (Sao Paulo). 2011;9(3):332-6. doi: 10.1590/S167945082011AO2002. [PubMed: 26761101].
2. Stephens RS, Sanchez-Pescador R, Wagar EA, Inouye C, Urdea MS. Diversity of Chlamydia trachomatis major outer membrane protein genes. J Bacteriol. 1987;169(9):3879-85. doi: 10.1128/jb.169.9.38793885.1987. [PubMed:3040664]. [PubMed Central: PMC213681].

3. World Health Organization. More than 1 million new curable sexually transmitted infections every day. Geneva, Switzerland: World Health Organization; 2019. Available from: https://www.who.int/news/item/06-06-2019-more-than-1million-new-curable-sexually-transmitted-infections-every-day.

4. Ostergaard O, Follmann F, Olsen AW, Heegaard NH, Andersen P, Rosenkrands I. Quantitative protein profiling of chlamydia trachomatis growth forms reveals defense strategies against tryptophan starvation. Mol Cell Proteomics. 2016;15(12):3540-50. doi: 10.1074/mcp.M116.061986. [PubMed: 27784728]. [PubMed Central: PMC5141270].

5. Eslami G, Goudarzi H, Taheripanah R, Taheri S, Fallah F, Moazzami B, et al. Chlamydia trachomatis detection by nested-PCR method on females referred to medical centers of Tehran, Iran. Arch Clin Infect Dis. 2012;7(4):124-7. doi: 10.5812/archcid.15087.

6. Afrasiabi S, Moniri R, Samimi M, Khorshidi A, Mousavi SG. The prevalence of endocervical chlamydia trachomatis infection among young females in Kashan, Iran. Jundishapur J Microbiol. 2015;8(4). e15576. doi: 10.5812/jjm.8(4)2015.15576. [PubMed: 26034530]. [PubMed Central: PMC4449842].

7. Bakhtiari A, Firoozjahi A. Chlamydia trachomatis infection in women attending health centres in Babol: Prevalence and risk factors. East Mediterr Health J. 2007;13(5):1124-31. doi: 10.26719/2007.13.5.1124. [PubMed: 18290406].

8. Jadranin Z, Ristanovic E, Atanasievska S, Dedic G, Sipetic-Grujicic S, Bokonjic D, et al. Prevalence and risk factors of chlamydia trachomatis genital infection among military personnel of the armed forces of Serbia: A cross-sectional study. Vojnosanit Pregl. 2019;76(2):168-74. doi: 10.2298/vsp170424088j.

9. Barton J, Braxton J, Davis D, de Voux A, Flagg E, Grier L, et al. Sexually transmitted disease surveillance. Atlanta, Georgia: Centers for Disease Control and Prevention; 2015.

10. Frej-Madrzak M, Grybos A, Grybos M, Teryks-Wolyniec D, JamaKmiecik A, Sarowska J, et al. PCR diagnostics of Chlamydia trachomatis in asymptomatic infection by women. Ginekol Pol. 2018;89(3):115-9. doi: 10.5603/GP.a2018.0020. [PubMed: 29664545].

11. Menon S, Timms P, Allan JA, Alexander K, Rombauts L, Horner $P$, et al. Human and pathogen factors associated with Chlamydia trachomatis-related infertility in women. Clin Microbiol Rev. 2015;28(4):969-85. doi: 10.1128/CMR.00035-15. [PubMed: 26310245]. [PubMed Central: PMC4548260].

12. Tadesse E, Teshome M, Amsalu A, Shimelis T. Genital Chlamydia trachomatis infection among women of reproductive age attending the gynecology clinic of Hawassa University Referral Hospital, Southern Ethiopia. PLoS One. 2016;11(12). e0168580. doi: 10.1371/journal.pone.0168580. [PubMed: 28006003]. [PubMed Central: PMC5178988].

13. Malhotra M, Sood S, Mukherjee A, Muralidhar S, Bala M. Genital Chlamydia trachomatis: An update. Indian J Med Res. 2013;138(3):30316. [PubMed: 24135174]. [PubMed Central: PMC3818592].

14. Haghighi Hasanabad M, Mohammadzadeh M, Bahador A, Fazel N, Rakhshani H, Majnooni A. Prevalence of Chlamydia trachomatis and Mycoplasma genitalium in pregnant women of Sabzevar-Iran. Iran J Microbiol. 2011;3(3):123-8. [PubMed: 22347594]. [PubMed Central: PMC3279818].

15. Chamani-Tabriz L, Tehrani MJ, Akhondi MM, Mosavi-Jarrahi A, Zeraati $\mathrm{H}$, Ghasemi J, et al. Chlamydia trachomatis prevalence in Iranian women attending obstetrics and gynaecology clinics. Pak J Biol Sci. 2007;10(24):4490-4. doi: 10.3923/pjbs.2007.4490.4494. [PubMed: 19093517]. 
16. Patel AL, Sachdev D, Nagpal P, Chaudhry U, Sonkar SC, Mendiratta SL, et al. Prevalence of Chlamydia infection among women visiting a gynaecology outpatient department: Evaluation of an in-house PCR assay for detection of Chlamydia trachomatis. Ann Clin Microbiol Antimicrob. 2010;9:24. doi: 10.1186/1476-0711-9-24. [PubMed: 20822551]. [PubMed Central: PMC2944303].

17. Al-Ramahi M, Mahafzah A, Saleh S, Fram K. Prevalence of Chlamydia trachomatis infection in infertile women at a university hospital in Jordan. East Mediterr Health J. 2008;14(5):1148-54. [PubMed: 19161088].

18. Bello S, Tunau K, Nasir S, Yahaya M, Panti A, Hassan M, et al. Prevalence of genital Chlamydia trachomatis infection among patients attending a gynecological clinic in a tertiary hospital. Sahel Medical Journal. 2019;22(4):188. doi:10.4103/smj.smj_64_18.
19. Falah F, Kazemi B, Goudarzi H, Badami N, Doustdari F, Ehteda A, et al Detection of Chlamydia trachomatis from urine specimens by PCR in women with cervicitis. Iranian J Publ Health. 2005;34(2):20-6.

20. Hocking JS, Willis J, Tabrizi S, Fairley CK, Garland SM, Hellard M. A chlamydia prevalence survey of young women living in Melbourne, Victoria. Sex Health. 2006;3(4):235-40. doi: 10.1071/sh06033. [PubMed: 17112433].

21. Kayiira A, Zaake D, Lwetabe MW, Sekweyama P. Impact of genital Chlamydia trachomatis infection on reproductive outcomes among infertile women undergoing tubal flushing: A retrospective cohort at a fertility centre in Uganda. Fertil Res Pract. 2019;5:16. doi: 10.1186/s40738-019-0069-5. [PubMed: 31890236]. [PubMed Central: PMC6909488]. 